\title{
INFERENCE ON A SET OF STATISTICAL MODELS
}

\author{
Yuzo Hosoya*
}

\begin{abstract}
The paper outlines some aspects related to statistical model selection, focusing in particular on inference conducted in the presence of a finite set of parametric models. The point the paper emphasizes is that the basic approaches such as testing, point estimation and confidence region estimation based on a single model are extensible under pertinent modification to inference on a set of models. They are, however, replaced by plural-model testing, 'point' model estimation and confidence-set construction of models.
\end{abstract}

Key words and phrases: AIC, CIC, confidence set of models, generalized likelihood ratio test, GIC, information criteria.

\section{Introduction}

The conventional paradigm of statistical inference based on the pair of a well-defined statistical model and a set of data does not correspond to practice; but pairing a plural set of alternative models to a data-set would often be more appropriate. In the presence of multiple composite hypotheses, there are somewhat different approaches such as nonparametric or robust methods, or decision-theoretic model selection methods. These three approaches are by no means clearly separated disciplines with distinct boundaries between them. To distinguish the proper model selection issue from the research interests of the former two approaches, we focus in the sequel on the situation where a data set is analyzed in the presence of a finite number of parametric models.

Suppose that the observation $Y^{(n)} \equiv\left(Y_{1}, \ldots, Y_{n}\right)$ is generated by a probability distribution whose density is given by $g_{n}\left(y^{(n)}\right)$ whereas the models are provided by the density defined on a nested sequence of parameter spaces $\Theta_{1}, \Theta_{2}, \ldots$, where if $j<k, \Theta_{j}$ is assumed embedded in $\Theta_{k}$. For such a nested structure of models, the basic concepts such as maximum likelihood estimation, sufficiency and ancillarity do not work effectively. The maximum likelihood for a parameter space cannot exceed the one for a properly including parameter space. A sufficient statistic defined for $\Theta_{k}$ remains sufficient for $\Theta_{j}$, but there is no guarantee that the statistic is minimal sufficient for it. Similarly an ancillary statistic for $\Theta_{j}$ is not necessarily ancillary for $\Theta_{k}$. There is no definitive inferential approach in the presence of such multiple parameter spaces. Even the assertion that the aim of statistical model selection is to find a parameter-parsimonious model is not necessarily justified. For instance, if we fit a regression model representing an economic behavior such as investment to data-sets of different countries, it is not necessarily wise to look for the simplest model. To elicit common features

\footnotetext{
Accepted September 12, 2007.

*Department of Economics, Meisei University, Hodokubo 2-1-1, Hino 191-8506, Japan. Email: yhosoya@econ.meisei-u.ac.jp
} 
and differences in investment behavior across different countries, a general model encompassing those different features would be more desirable.

This paper examines the decision-theoretic approach to model selection which was initiated by Akaike (1973). Section 2 characterizes his method as a decision-theoretic model selection based on the asymptotic approximation of the relative-entropy risk, extending it to the Gaussian stationary time-series model, the Box-Cox data transformations and a model of separate family of hypotheses. By means of a linear regression model, Section 3 compares in terms of exact small-sample properties the generalized information criterion (GIC) for different indices and also determines the opportunity-risk minimaxmizing GIC. Generalized information criteria with indices which are increasing functions of sample size have been proposed by Schwarz (1978), Akaike (1978) and Hannan and Rissanen (1982). The section shows that the maximum risk of a GIC estimate tends to infinity with increasing sample size if its index tends to infinity, whereas an estimate by GIC with a fixed index has a uniformly bounded risk. As for optimality, the AIC does not seem to have any definitive advantage over other GIC's in small-sample situations. Even in large samples any general superiority of AIC over other choice criteria does not seem to have been established. Although Shibata (1981) claims an asymptotic efficiency of AIC, his result is rather more appropriately termed the asymptotic admissibility (or asymptotic local efficiency) of AIC. Besides what he established is not inherent only to the AIC; a similar result is observed to hold for other selection methods if they are based on an appropriately estimated risk; see Hosoya (2002, pp. 174-9) for a detailed discussion. Section 4 discusses construction of confidence sets of models and consistent information criteria, and Section 5 is for concluding remarks.

\section{Estimation of risk}

The AIC is heuristically derived like this. Let $Y_{1}, \ldots, Y_{n}$ be a set of random variables which has the joint cumulative distribution function $G_{n}\left(y^{(n)}\right)$. Let $f_{n}\left(y^{(n)} \mid \theta\right)$ be a probability density function on the real $n$-space $R^{n}$ and $\theta$ be a parameter such that $\theta^{(p)}=\left(\theta_{1}, \ldots, \theta_{p}\right) \in \Theta^{(p)}$ an open-subset of $R^{p}$. The relative-entropy loss of the use of $f_{n}\left(\cdot \mid \theta^{(p)}\right)$ for modeling $Y^{(n)} \equiv\left(Y_{1}, \ldots, Y_{n}\right)$ is given by

$$
L_{n}\left(\theta^{(p)}, f_{n}\right)=-\int_{R^{n}} \frac{1}{n} \log f_{n}\left(y^{(n)} \mid \theta^{(p)}\right) d G_{n}\left(y^{(n)}\right),
$$

from which the cross-entropy risk of $f\left(\cdot \mid \tilde{\theta}_{n}^{(p)}\left(Y^{(n)}\right)\right)$ for an estimate $\tilde{\theta}_{n}^{(p)}$ is defined by

$$
R_{n}\left(\tilde{\theta}_{n}^{(p)}, f_{n}\right)=-\int_{R^{n}}\left\{\int_{R^{n}} \frac{1}{n} \log f_{n}\left(y^{(n)} \mid \tilde{\theta}_{n}^{(p)}\left(z^{(n)}\right)\right) d G_{n}\left(y^{(n)}\right)\right\} d G_{n}\left(z^{(n)}\right) .
$$

Assume that there is a unique $\theta^{0}$ such that $L_{n}\left(\theta^{0}, f_{n}\right)=\min _{\theta \in \Theta(p)} L_{n}\left(\theta, f_{n}\right)$ and let $\hat{\theta}_{n}^{(p)}$ be the maximum-likelihood estimate based on the model $f_{n}\left(\cdot \mid \theta^{(p)}\right)$. An approximately unbiased estimate of the cross-entropy risk of $\hat{\theta}_{n}^{(p)}$ is constructed by 
comparison of the formal asymptotic expansion of the risk with the expectation of the log-likelihood evaluated at $\hat{\theta}_{n}^{(p)}$. Set

$$
K_{n}\left(\hat{\theta}_{n}^{(p)}, f_{n}\right)=\sum_{j=1}^{p} \sum_{k=1}^{p} E\left\{\left(\hat{\theta}_{n, j}^{(p)}-\theta_{j}^{0}\right)\left(\hat{\theta}_{n, k}^{(p)}-\theta_{k}^{0}\right)\right\} \int_{R^{n}} \frac{\partial^{2} \log f_{n}\left(y^{(n)} \mid \theta^{0}\right)}{n \partial \theta_{j} \partial \theta_{k}} d G_{n}\left(y^{(n)}\right)
$$

then the quantity

$$
-\frac{1}{n} \log f_{n}\left(Y^{(n)} \mid \hat{\theta}_{n}^{(p)}\left(Y^{(n)}\right)\right)+K_{n}\left(\hat{\theta}_{n}^{(p)}, f_{n}\right)
$$

has the expectation whose formal expansion coincides with that of $R_{n}\left(\hat{\theta}_{n}^{(p)}, f_{n}\right)$ up to the order $O\left(n^{-1}\right)$. If $G_{n}$ has the density $f\left(\cdot \mid \theta^{0}\right), \theta^{0} \in \Theta^{(p)}$; then it follows from the information equality that $K_{n}\left(\hat{\theta}_{n}^{(p)}, f_{n}\right)=p / n+o\left(n^{-1}\right)$ and the quantity

$$
l_{n}\left(\hat{\theta}_{n}^{(p)}\right)=-\frac{1}{n} \log f_{n}\left(Y^{(n)} \mid \hat{\theta}_{n}^{(p)}\left(Y^{(n)}\right)\right)+\frac{p}{n},
$$

which Akaike proposes to use for model choice. Though $l_{n}\left(\hat{\theta}_{n}^{(p)}\right)$ does not approximate $R_{n}\left(\hat{\theta}_{n}^{(p)}, f_{n}\right)$ itself, the difference $l_{n}\left(\hat{\theta}_{n}^{(q)}\right)-l_{n}\left(\hat{\theta}_{n}^{(p)}\right)$ turns out to be an unbiased (up to $O\left(n^{-1}\right)$ ) estimate of $R_{n}\left(\hat{\theta}_{n}^{(q)}\right)-R_{n}\left(\hat{\theta}_{n}^{(p)}\right)$ for many practically important cases such as linear regression models or autoregressive models, where $\hat{\theta}_{n}^{(q)}$ and $\hat{\theta}_{n}^{(p)}$ are the maximum-likelihood estimates of $\theta$ when the parameter space of $f(\cdot \mid \theta)$ is restricted to the $q$-dimensional or $p$-dimensional parameter spaces $\Theta^{(q)}$ and $\Theta^{(p)}$. For cases where the information equality does not hold, a consistent estimate of $K_{n}\left(\hat{\theta}_{n}^{(p)}, f_{n}\right)$ would be employed to construct an approximately unbiased estimate of $R_{n}$. For instance, for cases where $Y_{1}, \ldots, Y_{n}$ are independent and $f_{n}\left(y^{(n)} \mid \theta^{(p)}\right)=f_{n}\left(y_{1} \mid \theta^{(p)}\right) \cdots f_{n}\left(y_{n} \mid \theta^{(p)}\right)$, Takeuchi (1976) proposed the estimate

$$
\tilde{K}_{n}\left(\hat{\theta}_{n}^{(p)}, f_{n}\right)=-\operatorname{trace} T_{n}^{-1} S_{n} / n
$$

where $T_{n}$ and $S_{n}$ are $p \times p$ matrices whose $(i, j)$ elements are respectively given by

$$
\sum_{t=1}^{n} \frac{\partial^{2} \log f_{n}\left(Y_{t}, \hat{\theta}_{n}^{(p)}\right)}{\partial \theta_{i} \partial \theta_{j}} \quad \text { and } \quad \sum_{t=1}^{n} \frac{\partial \log f_{n}\left(Y_{t} \mid \hat{\theta}_{n}^{(p)}\right)}{\partial \theta_{i}} \frac{\partial \log f_{n}\left(Y_{t} \mid \hat{\theta}_{n}^{(p)}\right)}{\partial \theta_{j}}
$$

However, the replacement of $p / n$ by $\tilde{K}_{n}\left(\hat{\theta}_{n}, f_{n}\right)$ would not have substantial effect as far as selection among nested models is concerned, since in comparison of $\Theta^{(p)}$ and $\Theta^{(q)}(p<q)$ for instance if $\Theta^{(p)}$ is true, $p / n$ is the correct estimate whereas if $\Theta^{(q)}$ is correct the likelihood ratio dominates the penalty difference of either $(q-p) / n$ or $\tilde{K}_{n}\left(\hat{\theta}_{n}^{(q)}, f_{n}\right)-\tilde{K}_{n}\left(\tilde{\theta}_{n}^{(p)}, f_{n}\right)$.

Example 2.1. Suppose that $Y_{1}, \ldots, Y_{n}$ are random variables generated from a Gaussian mean zero stationary process $\left\{Y_{t}: t \in \mathbb{Z}\right\}$ and that the spectral 
density model $g(\omega \mid \theta),|\omega| \leq \pi, \theta \in \Theta$, is chosen; whereas the true density of the process is $g(\omega)(|\omega| \leq \pi)$. Let $\hat{\theta}_{n}$ be the maximum likelihood estimate for the model $g(\omega \mid \theta)$ and define $\hat{M}_{g}$ and $\hat{V}_{g}$ respectively to be $p \times p$ matrices whose $(k, l)$ elements are

$$
\sum_{j=1}^{n}\left[\left(\frac{\partial^{2}}{\partial \theta_{k} \partial \theta_{l}} g\left(\omega_{j} \mid \theta\right)^{-1}\right) I_{n}\left(\omega_{j}\right)+\frac{\partial^{2}}{\partial \theta_{k} \partial \theta_{l}} \log g\left(\omega_{j} \mid \theta\right)\right]_{\theta=\hat{\theta}_{n}}
$$

and

$$
4 \pi \sum_{j=1}^{n}\left[\left(\frac{\partial}{\partial \theta_{k}} g\left(\omega_{j} \mid \theta\right)^{-1}\right)\left(\frac{\partial}{\partial \theta_{l}} g\left(\omega_{j} \mid \theta\right)^{-1}\right) I_{n}\left(\omega_{j}\right)^{2}\right]_{\theta=\hat{\theta}_{n}}
$$

where $\omega_{j}=-\pi+2(j-1) \pi / n$ and $I_{n}$ is the periodogram $I_{n}(\omega)=\sum_{t=1}^{n}\left|Y_{t} e^{i \omega j}\right|^{2} /$ $(2 \pi n)$. Then under a pertinent mixing condition for $\left\{Y_{t}\right\}$, the semiparametric quantity $\hat{K}_{n}\left(\hat{\theta}_{n}, g\right)=$ trace $\hat{M}_{g}^{-1} \hat{V}_{g} / n$ has the asymptotic expectation whose formal expansion coincides with $K_{n}\left(\hat{\theta}_{n}, g\right)$ up to order $O(1)$; see Hosoya and Taniguchi (1982) for the asymptotics involved and see also Hosoya (1984).

Example 2.2. Let $Y_{1}, \ldots, Y_{n}$ be independently, identically distributed positive random variables and consider the choice between two probability densities $f$ and $g$ given as $f(y, \alpha)=\exp \left[-\left(\log y-\alpha_{1}\right)^{2} / 2 \alpha_{2}\right] /\left\{y\left(2 \pi \alpha_{2}\right)^{1 / 2}\right\}$ and $g(y, \beta)=\beta^{-1} \exp (-y / \beta)$ for the model of the $Y_{j}$ 's (see Cox (1961)). Let $l_{1}=$ $\sum_{j=1}^{n} \log Y_{j} / n, l_{k}=\sum_{j=1}^{n}\left(\log Y_{j}-l_{1}\right)^{k} / n, k=2,4 ; m_{1}=\sum_{j=1}^{n} Y_{j} / n, m_{2}=$ $\sum_{j=1}^{n}\left(Y_{j}-m_{1}\right)^{2} / n$. Then, we have $\tilde{K}_{n}\left(\hat{\alpha}_{1}, f_{n}\right)=\left(1+l_{4} / l_{2}^{2}\right) / 2$ and $\tilde{K}_{n}\left(\hat{\beta}, g_{n}\right)=$ $m_{2} / m_{1}^{2}$, and consequently the choice rule based on the estimated relative-entropy risk is given as this: Choose the model $f(\cdot, \alpha)$ if

$$
-\sum_{j=1}^{n} \log f\left(Y_{j}, \hat{\alpha}\right)+\frac{1}{2}+\frac{1}{2} \frac{l_{4}}{l_{2}^{2}} \leq-\sum_{j=1}^{n} \log g\left(Y_{j}, \hat{\beta}\right)+\frac{m_{2}}{m_{1}^{2}},
$$

and choose $g(\cdot, \beta)$ otherwise, where $\hat{\alpha}$ and $\hat{\beta}$ are the maximum likelihood estimates under the respective models.

Example 2.3. Let $Y_{1}, \ldots, Y_{n}$ be independent, positive random variables having the probability density function indexed by $\lambda$ which is given as

$$
\begin{aligned}
& f_{n}^{\lambda}\left(y^{(n)}, \beta, \sigma^{2}\right) \\
& \quad=\frac{1}{(2 \pi)^{n / 2} \sigma^{n}} \exp \left\{-\sum_{j=1}^{n}\left(y_{j}^{[\lambda]}-\sum_{k=1}^{p} \beta_{k} x_{j k}\right)^{2} /\left(2 \sigma^{2}\right)\right\} \sum_{j=1}^{n} y_{j}^{\lambda-1}
\end{aligned}
$$

where $y^{[\lambda]}=\left(y^{\lambda}-1\right) / \lambda$ and $y^{[\lambda]}=\log y$ if $\lambda=0$; the design matrix $X=$ $\left\{x_{j k}: j=1, \ldots, n ; k=1, \ldots, p\right\}$ has rank $p$ (see Box and Cox (1964)). Let $\hat{\beta}_{\lambda j}$ and $\hat{\sigma}_{\lambda}^{2}$ be the maximum likelihood estimate of $\beta_{j}, \sigma^{2}$, let $\hat{\delta}_{\lambda}=\sum_{j=1}^{n}\left(Y_{j}^{[\lambda]}-\right.$ $\left.\sum_{k=1}^{p} \hat{\beta}_{\lambda k} x_{j k}\right)^{2} / n$ and denote by $T^{\lambda}$ the $p \times p$ matrix whose $(l, m)$ element is 
$\sum_{j=1}^{n}\left(Y_{j}^{[\lambda]}-\sum_{k=1}^{p} \hat{\beta}_{\lambda k} x_{j k}\right)^{2} x_{j l} x_{j m} / n$. Then by a straightforward calculation, we have

$$
\tilde{K}\left(\hat{\beta}_{\lambda}, \hat{\sigma}_{\lambda}^{2}, f_{n}^{\lambda}\right)=2 \hat{\sigma}_{\lambda}^{2} \operatorname{trace}\left\{\left(X^{\prime} X\right)^{-1} T^{\lambda}\right\} / n+\left\{\hat{\delta}_{\lambda} /\left(\hat{\sigma}_{\lambda}^{2}\right)^{2}-1\right\} /(2 n)
$$

Consequently $\lambda$ which minimizes

$$
\begin{aligned}
J_{n}(\lambda)= & -\frac{1}{2} \log \hat{\sigma}_{\lambda}^{2}+(\lambda-1) \sum_{j=1}^{n} \log Y_{j} / n \\
& +2 \hat{\sigma}_{\lambda}^{2} \operatorname{trace}\left\{\left(X^{\prime} X\right)^{-1} T^{\lambda}\right\} / n+\left\{\hat{\delta}_{\lambda} /\left(\hat{\sigma}_{\lambda}^{2}\right)^{2}-1\right\} /(2 n)
\end{aligned}
$$

will be selected instead of the direct maximum-likelihood estimation of $\lambda$; see Yao (2002) for a detailed simulation study on the small-sample performance of this model-selection criterion.

\section{Linear regression model and the generalized information criterion}

Suppose there are two alternative probability densities $f_{n}^{p}\left(y^{(n)} \mid \beta^{(p)}\right)$, $f_{n}^{p+1}\left(y^{(n)} \mid \beta^{(p+1)}\right)$ of random variables $Y_{1}, \ldots, Y_{n}$ where

$$
f_{n}^{p+1}\left(y^{(n)} \mid \beta^{(p+1)}\right)=\frac{1}{(\sqrt{2 \pi} \sigma)^{n}} \exp \left\{-\sum_{j=1}^{n}\left(y_{j}-\sum_{k=1}^{p+1} \beta_{k} x_{j k}\right)^{2} /\left(2 \sigma^{2}\right)\right\}
$$

and $f_{n}^{p}\left(y^{(n)} \mid \beta^{(p)}\right)=f_{n}^{p+1}\left(y^{(n)} \mid \beta^{(p)}, 0\right)$. This section investigates the behavior of GIC with respect to the selection between $f_{n}^{p}$ and $f_{n}^{p+1}$. The design matrix $X=\left\{x_{j k}: j=1, \ldots, n ; k=1, \ldots, p+1\right\}$ is assumed to satisfy $X^{\prime} X=I_{p+1}$ where $I_{p+1}$ is the identity matrix of order $p+1$ and $\sigma^{2}$ is assumed, at first, known. Let GIC with index $\alpha$ be defined by

$$
G I C_{\alpha}(q)=-\log f_{n}^{q}\left(Y_{1}, \ldots, Y_{n} \mid \hat{\beta}_{1}, \ldots, \hat{\beta}_{q}\right)+\alpha q, \quad q=p, \quad p+1,
$$

where $\hat{\beta}_{1}, \ldots, \hat{\beta}_{q}$ are the maximum-likelihood estimates and $\alpha$ is a nonnegative constant $\left(\alpha=1\right.$ for AIC); see Atkinson (1980). If $\operatorname{GIC}_{\alpha}(p) \leq G I C(p+1)$, equivalently if $\left|\hat{\beta}_{p+1}\right|^{2} \leq 2 \alpha \sigma^{2}$, the model $f_{n}^{p}$ is selected, and $f_{n}^{p+1}$ is selected otherwise. Define $\beta^{*}(\alpha)$ as a vector such that $\beta^{*}(\alpha)=\left(\hat{\beta}_{1}, \ldots, \hat{\beta}_{p}, 0\right)$ if $\left|\hat{\beta}_{p+1}\right|^{2} \leq$ $2 \alpha \sigma^{2} ; \beta^{*}(\alpha)=\left(\hat{\beta}_{1}, \ldots, \hat{\beta}_{p+1}\right)$, otherwise, and define the risk $S_{n}$ of $\beta^{*}(\alpha)$ as

$$
S_{n}\left(\beta^{*}(\alpha) \mid \beta_{p+1}\right)=E\left(\beta_{p+1}^{*}(\alpha)-\beta_{p+1}\right)^{2} / \sigma^{2} .
$$

Without loss of generality, the comparison of $G I C_{\alpha}$ for various $\alpha$ in terms of the relative-entropy risk is conducted in view of the risk $S_{n}$ only, because it is the only component of the former which varies with $\alpha$. Assume that the true model of $Y_{1}, \ldots, Y_{n}$ is either $f_{n}^{p}$ or $f_{n}^{p+1}$ [in case $\sigma$ is known this assumption is not essential and can be dispensed with by arguing only in terms of difference of 
risks]. Let $\lambda=\beta_{p+1} / \sigma$ and set $R(\alpha, \lambda)=S_{n}\left(\beta^{*}(\alpha) \mid \beta_{p+1}\right)$; then it follows from the definition of $S_{n}$ that

$$
\begin{aligned}
R(\alpha, \lambda)= & \frac{1}{\sigma^{2}} \int_{|\beta|^{2} \geq \alpha \sigma^{2}}\left(\beta-\beta_{p+1}\right)^{2} \frac{1}{\sqrt{2 \pi} \sigma} \exp \left\{-\frac{\left(\beta-\beta_{p+1}\right)^{2}}{2 \sigma^{2}}\right\} d \beta \\
& +\lambda^{2} \int_{|\beta|^{2} \leq \alpha \sigma^{2}} \frac{1}{\sqrt{2 \pi} \sigma} \exp \left\{-\frac{\left(\beta-\beta_{p+1}\right)^{2}}{2 \sigma^{2}}\right\} d \beta \\
= & 1+\left(\lambda^{2}-1\right)\{\Phi(\sqrt{\alpha}-\lambda)-\Phi(-\sqrt{\alpha}-\lambda)\}+(\sqrt{\alpha}-\lambda) \phi(\sqrt{\alpha}-\lambda) \\
& +(\sqrt{\alpha}+\lambda) \phi(\sqrt{\alpha}+\lambda)
\end{aligned}
$$

where $\phi(x)=\frac{1}{\sqrt{2 \pi}} \exp \left(-x^{2} / 2\right)$ and $\Phi(y)=\int_{-\infty}^{y} \phi(x) d x$. Define $R^{*}(\lambda)$ by $R^{*}(\lambda)=$ in $f_{\alpha} R(\alpha, \lambda)$; it follows easily from (3.1) that

$$
R^{*}(\lambda)=\lambda^{2} \quad \text { if }|\lambda| \leq 1 \quad \text { and } \quad R^{*}(\lambda)=1 \quad \text { otherwise. }
$$

The minimax estimate for the risk $R$ above is given by $\beta^{*}(0)$, whereas the minimax estimate $\beta^{*}\left(\alpha^{*}\right)$ for the opportunity risk $R(\alpha, \lambda)-R^{*}(\lambda)$ is given by GIC for an index $\alpha^{*}$. Let $\lambda^{*}$ be a value maximizing $R\left(\alpha^{*}, \lambda\right)-R^{*}(\lambda)$; then $\left(\alpha^{*}, \lambda^{*}\right)$ is not a saddle point of $R(\alpha, \lambda)-R^{*}(\lambda)$, but the minimax $\alpha^{*}$ is provided as the minimizing $\alpha$ of

$$
R^{\dagger}(\alpha) \equiv \max _{|\lambda|>1}\left\{R(\alpha, \lambda)-R^{*}(\lambda)\right\}
$$

and numerically it is determined as $0.93738840 \leq \alpha^{*} \leq 0.937388842$ for which we have $0.598799193 \leq R\left(\alpha^{*}, \lambda^{*}\right)-R^{*}\left(\lambda^{*}\right) \leq 0.598799195 . R^{\dagger}(\alpha)$ is a decreasing function of $\alpha$ and $\max _{\lambda} R(\alpha, \lambda)$ is an increasing function of $\alpha$, and every choice of $\alpha$ is seen to give an admissible selection method. Figure 1 gives the graphs of $R$ for $\alpha=0.5, \alpha^{*}(\operatorname{minimax}), 1(A I C)$; see Sawa and Hiromatu (1969) for a related, but somewhat different approach.

To investigate the asymptotic behavior of $G I C_{\alpha_{n}}$ as $n$ tends to infinity when $\alpha_{n}$ may vary with $n$, it is convenient to assume that the variance $\sigma^{2}=\sigma_{n}^{2}$ is a function of $n$ and $\lim _{n \rightarrow \infty} \sigma_{n}^{2}=0$, since the column vectors of the design matrix $X$ are standardized by assumption. Then, it is easily seen that the conditions $\lim _{n \rightarrow \infty} \alpha_{n}=\infty$ and $\lim _{n \rightarrow \infty} \alpha_{n} \sigma_{n}=0$ are necessary and sufficient for the relationship

$$
\lim _{n \rightarrow \infty} S_{n}\left(\beta^{*}\left(\alpha_{n}\right) \mid \beta_{p+1}\right) / \inf _{\alpha} S_{n}\left(\beta^{*}(\alpha) \mid \beta_{p+1}\right)=1
$$

to hold for any fixed $\beta_{p+1}$. But the apparent efficiency of (3.3) depends critically on the pointwise convergence, and in terms of uniform convergence, it turns out that

$$
\lim _{n \rightarrow \infty} \sup _{\beta_{p+1}} S_{n}\left(\beta^{*}\left(\alpha_{n}\right) \mid \beta_{p+1}\right)=\infty
$$

for any sequence $\alpha_{n}$ which tends to infinity, as is seen by setting $\lambda=\alpha_{n}-1$ in (3.1). On the other hand we have for example $\sup _{\beta_{p+1}} S_{n}\left(\beta^{*}\left(\alpha^{*}\right) \mid \beta_{p+1}\right)<1.6$ for all $n$. 


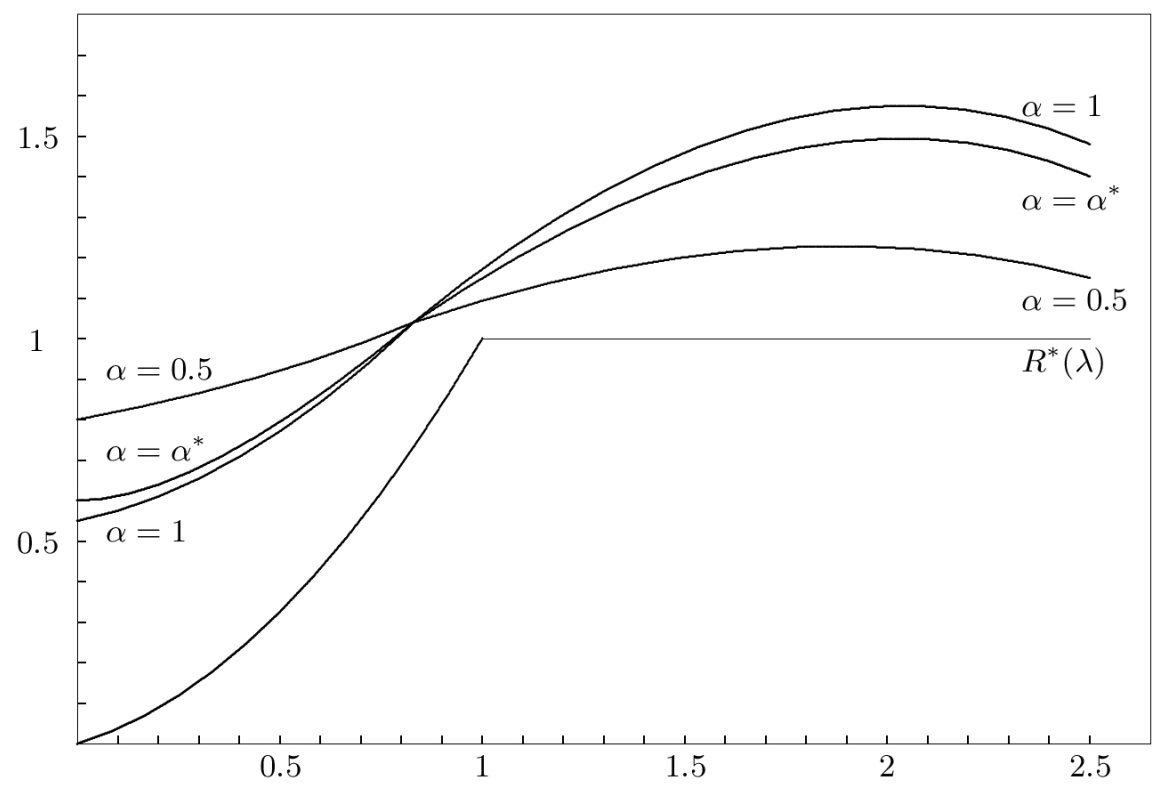

Figure 1. The graph of the risk $R(\alpha, \lambda)$ for $\alpha=0.5, \alpha^{*}, 1$, and $R^{*}(\lambda)$.

For the case where $\sigma$ is unknown, the GIC is defined as

$$
G I C_{\alpha}^{*}(q)=\sum_{j=1}^{n}\left(Y_{t}-\sum_{k=0}^{q} \hat{\beta}_{k} x_{j k}\right)^{2} / 2+\alpha q \hat{\sigma}^{2}, \quad q=p, \quad p+1
$$

where $\hat{\sigma}^{2}=\sum_{t=1}^{n}\left(Y_{t}-\sum_{j=0}^{q} \hat{\beta}_{j} x_{t j}\right)^{2} /(n-p-1)$. Define an estimate $\beta^{\dagger}(\alpha)$ as $\beta^{\dagger}(\alpha)=\left(\hat{\beta}_{1}, \ldots, \hat{\beta}_{p+1}\right)$ if $\operatorname{GIC}_{\alpha}^{*}(p+1) \leq G I C_{\alpha}^{*}(p)$ and $\beta^{\dagger}(\alpha)=\left(\hat{\beta}_{1}, \ldots, \hat{\beta}_{p}, 0\right)$, otherwise. As in the preceding argument, define the risk $S_{n}$ of $\beta^{\dagger}(\alpha)$ as

$$
S_{n}\left(\beta^{\dagger}(\alpha) \mid \beta_{p+1}\right)=E\left(\beta_{p+1}^{\dagger}(\alpha)-\beta_{p+1}\right)^{2} / \sigma^{2},
$$

and assume as before that the true model of $Y_{1}, \ldots, Y_{n}$ is either $f_{n}^{p}$ or $f_{n}^{p+1}$. Then since $(n-p-1) \hat{\sigma}^{2} / \sigma^{2}$ has the $\chi^{2}$ distribution with degrees of freedom $\nu=n-p-1$ and is independent of $\hat{\beta}_{p+1}$, it follows from (3.1) that

$$
\begin{aligned}
& S_{n}\left(\beta^{\dagger}(\alpha) \mid \beta_{p+1}\right) \equiv S(\alpha, \lambda, \nu)=\int_{0}^{\infty}\left[1-\left(1-\lambda^{2}\right)\{\Phi(\sqrt{\alpha} y-\lambda)-\Phi(-\sqrt{\alpha} y-\lambda)\}\right. \\
& \quad+\frac{1}{\sqrt{2 \pi}}(\sqrt{\alpha} y-\lambda) \exp \left\{-(\sqrt{\alpha} y-\lambda)^{2} / 2\right\} \\
&\left.\quad+\frac{1}{\sqrt{2 \pi}}(\sqrt{\alpha} y+\lambda) \exp \left\{-(\sqrt{\alpha} y+\lambda)^{2} / 2\right\}\right] \\
& . C(\nu) y^{\nu-1} \exp \left(-\nu y^{2} / 2\right) d y,
\end{aligned}
$$


where $C(\nu)=\nu^{\nu / 2} /\left(2^{(\nu / 2)-1} \Gamma(\nu / 2)\right)$. Set

$$
\begin{aligned}
\gamma(\alpha, \nu, \lambda, l)= & \frac{C(\nu)}{\sqrt{2 \pi}} \int_{0}^{\infty} y^{l} \exp \left\{-\frac{(\sqrt{\alpha} y-\lambda)^{2}}{2}\right\} \exp \left\{-\frac{\nu y^{2}}{2}\right\} d y \\
= & \frac{C(\nu)}{\sqrt{2 \pi}} \exp \left\{-\frac{\nu \lambda^{2}}{2(\alpha+\nu)}\right\} \sum_{j=0}^{l}{ }_{l} C_{j} \frac{2^{(j-1) / 2}(\sqrt{\alpha} \lambda)^{l-j}}{(\alpha+\nu)^{1 / 2+l-(j / 2)}} \\
& \cdot\left\{\Gamma\left(\infty \mid \frac{j+1}{2}\right)+k(j) \Gamma\left(\frac{\alpha \lambda^{2}}{2(\alpha+\nu)} \mid \frac{j+1}{2}\right)\right\}
\end{aligned}
$$

where $k(j)=(-1)^{j}$ if $\lambda \geq 0$ and $k(j)=-1$ if $\lambda<0$ and $\Gamma(\infty \mid s)$ and $\Gamma(x \mid s)$ denote respectively the $\Gamma$ function and the incomplete $\Gamma$ function with index $s$. Let $\mu$ be a positive integer and suppose $\nu=2 \mu$. Then by repeated application of integration by parts, $S(\alpha, \lambda, 2 \mu)$ is evaluated as follows:

$$
\begin{aligned}
S(\alpha, \lambda, 2 \mu)=1 & +\sqrt{\alpha}\{\gamma(\alpha, \mu, \lambda, 2 \mu)+\gamma(\alpha, \mu,-\lambda, 2 \mu)\} \\
& -\lambda \gamma(\alpha, \mu, \lambda, 2 \mu-1)+\lambda \gamma(\alpha, \mu,-\lambda, 2 \mu-1) \\
& +\frac{\left(\lambda^{2}-1\right) \sqrt{\alpha}}{\nu} \sum_{j=0}^{\mu-1}\left(\prod_{l=0}^{j} \frac{\nu-2 l}{\nu}\right) \\
& \times\{\gamma(\alpha, \mu, \lambda, \nu-2 j-2)+\gamma(\alpha, \mu,-\lambda, \nu-2 j-2)\} .
\end{aligned}
$$

As in (3.2), set $S^{*}(\lambda, 2 \mu)=\inf _{\alpha} S(\alpha, \lambda, 2 \mu)$; then $S^{*}(\lambda, 2 \mu)=\lambda^{2}$ if $|\lambda| \leq 1$ and $S^{*}(\lambda, 2 \mu)=1$, otherwise. Denote by $\alpha^{*}$ the value of $\alpha$ which minimizes the maximum opportunity risk $M(\alpha, \mu)=\sup _{\lambda}\left\{S(\alpha, \lambda, 2 \mu)-S^{*}(\lambda, \mu)\right\}$. Table 1 exhibits $\alpha^{*}$ 's and $M(\alpha, 2 \mu)$ for $\alpha=\alpha^{*}, 1$ for several $\nu$ 's.

When $\nu=2 \mu-1$, the function $S$ is not represented as a linear combination only of $\Gamma$ and incomplete $\Gamma$ functions, but instead it is expressed as a series involving $F$ distributions. Let $Z$ be a standard normal random variable and $W$ be a $\chi^{2}$ random variable with degree of freedom $\nu$ and suppose $Z$ and $W$ are independent; then we have

$$
\begin{aligned}
& \operatorname{Pr}\left\{(Z+\lambda)^{2} /(W / \nu) \leq \alpha\right\} \\
& \quad=C(\nu) \int_{0}^{\infty}\{\Phi(\sqrt{\alpha} y-\lambda)-\Phi(-\sqrt{\alpha} y-\lambda)\} y^{\nu-1} \exp \left(-\nu y^{2} / 2\right) d y .
\end{aligned}
$$

Since $(Z+\lambda)^{2} /(W / \nu)$ is distributed as a non-central $F$-random variable with degrees of freedom $1,2 \mu-1$ and with non-central parameter $\lambda^{2} / 2$, it follows from (3.4) that

$$
\begin{aligned}
S(\alpha, \lambda, 2 \mu-1)= & +\sqrt{\alpha}\{\gamma(\alpha, \nu, \lambda, 2 \mu-1)+\gamma(\alpha, \nu,-\lambda, 2 \mu-1)\} \\
& -\gamma(\alpha, \nu, \lambda, 2 \mu-2)+\gamma(\alpha, \nu,-\lambda, 2 \mu-2) \\
& +\left(\lambda^{2}-1\right) \sum_{i=0}^{\infty}\left(\frac{1}{1+2 i}\right)^{((2 i+1) / 2)-1} \\
& \cdot \frac{\left(\lambda^{2} / 2\right)^{i} \exp \left(-\lambda^{2} / 2\right)}{i !} F_{1+2 i, 2 \mu-1}\left(\frac{2 \mu-1}{2 i+1} \alpha\right)
\end{aligned}
$$


Table 1. The minimax criterion and the maximum risks.

\begin{tabular}{rccc}
\hline \multicolumn{1}{c}{$\nu$} & $\alpha^{*}$ & $M\left(\alpha^{*}, \nu\right)$ & $M(1, \nu)$ \\
\hline 2 & 0.986 & $0.650(2.154)$ & $0.661(2.161)$ \\
4 & 0.961 & $0.628(2.009)$ & $0.658(2.036)$ \\
6 & 0.953 & $0.619(1.970)$ & $0.656(1.988)$ \\
8 & 0.949 & $0.614(1.945)$ & $0.655(1.963)$ \\
10 & 0.947 & $0.611(1.929)$ & $0.655(1.950)$ \\
20 & 0.942 & $0.605(1.896)$ & $0.653(1.916)$ \\
30 & 0.941 & $0.603(1.885)$ & $0.652(1.904)$ \\
40 & 0.940 & $0.602(1.880)$ & $0.652(1.899)$ \\
50 & 0.939 & $0.602(1.878)$ & $0.651(1.898)$ \\
$\infty$ & 0.937 & $0.599(1.864)$ & $0.651(1.883)$ \\
\hline
\end{tabular}

The figures in parentheses indicate the locations of the maximizing $\lambda$ (the other maximum is attained at the origin for $\alpha=\alpha^{*}$ ).

where $F_{m, n}(x)$ is the cumulative distribution function of the $F$-statistic with degrees of freedom $(m, n)$.

\section{Confidence sets of models}

Suppose that the joint probability density of a random vector $Y^{(n)}=\left(Y_{1}, \ldots\right.$, $\left.Y_{n}\right)$ is given by $f\left(y^{(n)} \mid \theta\right)$ and that the parameter spaces to which $\theta$ belongs have a nesting structure such that the $(p+1)$ parameter spaces have the inclusion relations

$$
\Theta^{\left(q_{0}\right)} \subset \Theta^{\left(q_{1}\right)} \subset \Theta^{\left(q_{2}\right)} \subset \cdots \subset \Theta^{\left(q_{p}\right)}
$$

where we assume that $\Theta^{\left(q_{i}\right)}$ is $q_{i}$-dimensional and $q_{0}<q_{1}<\cdots<q_{p}$. The hypothesis $H_{i}$ implies that $\theta \in \Theta^{\left(q_{i}\right)}$ and $\theta \notin \Theta^{\left(q_{i-1}\right)}$. Consequently we consider the situation where the model selection issue is reduced to the question of which $\Theta^{\left(q_{i}\right)}$ the true parameter $\theta$ belongs to. Consider then testing each $H_{i}$ against the alternative hypotheses $H_{i+1}, \ldots, H_{p}$ for significance level $\alpha$ and for $i=0, \ldots, p-$ 1. Let $\tilde{I}$ be the set constituted of those hypotheses not rejected by this test and $H_{p}$. Suppose that $H_{i_{0}}$ is the true model. Then since $\operatorname{Pr}\left\{H_{i_{0}} \in \tilde{I} \mid H_{i_{0}}\right\}=1-\alpha$ for $0 \leq i_{0} \leq p-1$, whereas $\operatorname{Pr}\left\{H_{p} \in \tilde{H} \mid H_{p}\right\}=1$ for the case $i_{0}=p, \tilde{I}$ is a confidence set of confidence level $(1-\alpha)$. Let $\tilde{i}$ be the minimal $i$ of the hypotheses $H_{i}$ belonging to $\tilde{I}$, then we have

$$
\operatorname{Pr}\left\{\tilde{i} \leq i_{0} \mid H_{i_{0}}\right\} \geq 1-\alpha, \quad i_{0}=0,1, \ldots, p .
$$

In other words, the set of models $\left\{H_{i}: i \geq \tilde{i}\right\}$ gives a confidence set of at least $(1-\alpha)$ confidence level. A way to test $H_{i}$ against $H_{i+1}, \ldots, H_{p}$ is to fix the critical values, $c_{i, j}, j=i+1, \ldots, p$, so that the log-likelihood ratios $L_{i, i+1}, L_{i, i+2}, \ldots, L_{i, p}$ have the same marginal (large sample) significance level for these critical values, at the same time making the overall significance level to be a given level $\alpha$, where 
the log-likelihood ratio $L_{i, j}$ is defined by

$$
L_{i, j}=2\left\{\max _{\theta \in \Theta^{\left(q_{j}\right)}} \log f\left(Y^{(n)} \mid \theta\right)-\max _{\theta \in \Theta^{\left(q_{i}\right)}} \log f\left(Y^{(n)} \mid \theta\right)\right\} .
$$

To be specific, the critical values $c_{i, j}$ are determined by $\operatorname{Pr}\left\{L_{i, j}>c_{i, j} \mid H_{i}\right\}=\gamma$ and $\operatorname{Pr}\left\{U_{j=i+1}^{p}\left\{L_{i, j}>c_{i, j}\right\} \mid H_{i}\right\}=\alpha$ and if $L_{i, j}>c_{i, j}$ for some $j,(i+1 \leq$ $j \leq p), H_{i}$ is rejected. A generalized likelihood-ratio test places every alternative hypothesis on an equal footing; see Hosoya $(1986,1989)$ for how the critical values $c_{i, j}$ are numerically determined. For related literature, see Terui (1990) for a simultaneous $F$ test to deal with nested regression models and also see Katayama (2006) for the non-central $\chi^{2}$ asymptotics of the GLR test and its use in model selection.

Consider in the sequel the situation where every $L_{i, j}, i+1 \leq j \leq p$, is asymptotically $\chi^{2}$-distributed if $H_{i}$ is true. Note that model $H_{\tilde{i}}$ in $(4.1)$ is the lower limit of the confidence set and that we have

$$
\lim _{n \rightarrow \infty} \operatorname{Pr}\left\{\tilde{i}=i_{0} \mid i_{0}\right\}=1
$$

by choosing the significance level $\alpha=\alpha_{n}$ in such a way that

$$
\text { (i) } \lim _{n \rightarrow \infty} \alpha_{n}=0, \quad \text { and } \quad \text { (ii) } \lim _{n \rightarrow \infty}\left(\log \alpha_{n}\right) / n=0 \text {. }
$$

Namely by suitable choice of the significance level $\alpha_{n}$, the corresponding lower limit of the confidence set can be a consistent estimator of the true model; see Hosoya (1989) for the mathematical proof.

By applying the preceding confidence-set approach, we can construct consistent-estimation information criteria. Let $h\left(q_{i}, n\right)$ be a positive number defined as follows. For the parameter space $\Theta^{\left(q_{i}\right)}, i=0, \ldots, p$, if $i<j, h\left(q_{i}, n\right)<h\left(q_{j}, n\right)$ and $\lim _{n \rightarrow \infty}\left\{h\left(q_{j}, n\right)-h\left(q_{i}, n\right)\right\}=\infty ; \lim _{n \rightarrow \infty} h\left(q_{i}, n\right) / n=0$. Then define an information criterion by

$$
C I C(i)=-\frac{1}{n} \log f_{n}\left(Y_{1}, \ldots, Y_{n} \mid \hat{\theta}_{n}^{\left(q_{i}\right)}\right)+\frac{h\left(q_{i}, n\right)}{n}
$$

see also Schwarz (1978) and Hannan and Rissanen (1982). Let $\hat{i}$ be the first $i$ which makes the CIC smallest, where $\hat{\theta}_{n}^{\left(q_{i}\right)}$ is the maximum likelihood estimator based on the parameter space $\Theta^{\left(q_{i}\right)}$. Suppose $i, j$ are two positive numbers such that $0 \leq i<j \leq p$; then since

$$
C I C(i)-C I C(j)=\frac{1}{n} L_{i, j}+\frac{1}{n}\left\{h\left(q_{i}, n\right)-h\left(q_{j}, n\right)\right\},
$$

the inequality $C I C(i) \geq(\leq) C I C(j)$ is equivalent to $L_{i, j} \geq(\leq)\left\{h\left(q_{j}, n\right)-\right.$ $\left.h\left(q_{i}, n\right)\right\} / n$. So we can regard the positive difference $\left\{h\left(q_{j}, n\right)-h\left(q_{i}, n\right)\right\} / n$ as a critical value of the likelihood-ratio test. The condition that the model $\hat{i}$ satisfies $C I C(\hat{i}) \leq C I C(k)$ for all hypothetical $k$ is equivalent to the condition that $\hat{i}$ 
is the first hypothesis accepted in the generalized likelihood ratio test. Therefore, by an argument paralleling (4.2) and in view of the asymptotic property of $h\left(q_{j}, n\right)-h\left(q_{i}, n\right), \hat{i}$ is proved to be a consistent estimator of $i_{0}$.

In model selection among a finite set, the criterion (2.1) or its generalized version

$$
-\frac{1}{n} \log f_{n}\left(Y^{(n)} \mid \hat{\theta}_{n}^{\left(q_{i}\right)}\left(Y^{(n)}\right)\right)+\frac{\alpha \cdot q_{i}}{n}, \quad \alpha>0,
$$

would be useful in determining an upper bound for a confidence set of models. In the situation where no upper limit is fixed in the model sequence $\Theta^{\left(q_{0}\right)}, \Theta^{\left(q_{1}\right)}, \ldots$ and a parameter space can be of arbitrarily high dimension, the use of an information criterion to determine a model would be equivalent rather to fixing a degree of smoothness in nonparametric statistics.

\section{Concluding remarks}

The outcome of an empirical analysis crucially depends upon the choice of the statistical model on which inference is conducted. Data sets are provided by experiments or surveys, whereas a model is chosen by the analyst. Based on his own experience of empirical modelling, Klein (1981) asserts the importance of model selection in improving macroeconomic forecasting accuracy. He is rather skeptical about the use of highly sophisticated statistical inference methods in improving economic predictions. In particular, he points out the superiority of multivariate time-series models which use lagged endogenous variables over simultaneous equation models. Making a clear distinction between the user and the producer of an empirical model, Granger (1999) claims that convenience is a priority for decision-making by the user in empirical model selection. According to him, models should not be compared in terms of a variety of academic qualities such as mathematical elegance or sophistication, but in terms of the pragmatic quality of how their outcomes help the user's decision making. In particular, he emphasizes the post-sample evaluation of outcomes of proposed models instead of the conventional model selection by in-sample testing or by in-sample information criteria. Sutton (2001) is pessimistic about economic theories and/or statistical model selection methods being able to pinpoint a unique empirical model which explains an empirical market performance, proposing instead robust characterization of the market performance which is stable against uncontrollable complexity of individual market structures. After all statistical methods alone would not be enough for pinpointing a pertinent model in empirical analysis situations. Good interdisciplinary communication and formal languages for that purpose need to be developed.

\section{Acknowledgements}

The research is partially supported by JSPS Grant (C) 19530190. I thank an anonymous referee for very helpful comments and suggestions. 


\section{REFERENCES}

Akaike, H. (1973). Information theory and an extension of the maximum likelihood principle, Second International Symposium on Information Theory (eds. B. N. Petrov and F. Cśaki), 267-281, Akadémia Kiado, Budapest.

Akaike, H. (1978). A Bayesian analysis of the minimum AIC procedure, Ann. Inst. Statist. Math., 30, 9-14.

Atkinson, A. C. (1980). A note on the generalized information criterion for choice of a model, Biometrika, 67, 413-418.

Box, G. E. P. and Cox, D. R. (1964). An analysis of transformations (with discussion), J. R. Statist. Soc., B26, 211-252.

Cox, D. R. (1961). Test of separate families of hypotheses, Proc. 4th Berkley Symp., 1, 105-123.

Granger, C. W. J. (1999). Empirical Modeling in Economics: Specification and Evaluation, Cambridge University Press, Cambridge.

Hannan, E. J. and Rissanen, J. (1982). Recursive estimation of mixed autoregressive moving average order, Biometrika, 60, 81-94.

Hosoya, Y. (1984). Information criteria and tests for time-series models, Time-Series Analysis: Theory and Practice 5 (ed. O. D. Anderson), 39-52, Elsevier Science, Amsterdam.

Hosoya, Y. (1986). A simultaneous test in the presence of nested alternative hypotheses, Journal of Applied Probability, 23A, 187-200.

Hosoya, Y. (1989). Hierarchical statistical models and a generalized likelihood ratio test, J. R. Statist. Soc., B51, 435-447.

Hosoya, Y. (2002). Statistical Evidence and its Interpretations: Enlarged edition, Makino Publishing Company, Tokyo (in Japanese).

Hosoya, Y. and Taniguchi, M. (1982). A central limit theorem for stationary processes and the parameter estimation of linear processes, Ann. Statist., 10, 132-153.

Katayama, N. (2006). On model-selection problems in terms of prediction mean squared error, Proc. Inst. Stat. Math., 54, 481-510 (in Japanese).

Klein, L. R. (1981). Econometric Models as Guides for Decision-Making, The Free Press, London.

Sawa, T. and Hiromatu, T. (1969). Minimax regret criterion for selection of explanatory variables, Review of Economics, 20, 316-321 (in Japanese).

Schwarz, G. (1978). Estimating the dimension of a model, Ann. Statist., 6, 461-464.

Shibata, R. (1981). An optimal selection of regression variables, Biometrika, 68, 45-54.

Sutton, J. (2001). Marshall's Tendencies: What Can Economists Know?, The MIT Press, Massachusetts.

Takeuchi, K. (1976). The distribution of information statistics and the criterion of goodness of fit of models, Mathematical Science, 153, 12-18 (in Japanese).

Terui, N. (1990). An F type small sample simultaneous test for nested linear regression models, Communications in Statistics: Theory and Methods, A19, 703-722.

Yao, F. (2002). Econometric Analysis of Nonstationary and Nonlinear Relationships (Kagawa University economic studies monograph series vol. 16), Shinzansha, Tokyo. 